\section{BACKGROUND, TESTING METHODS, AND LABORATORY APPROACHES TO SARS CORONAVIRUS-2 (SARS-COV-2) AND COVID19}

William D. Rawlinson ${ }^{1,2}$, Sebastiaan van $\mathrm{Hal}^{3}$, Malinna Yeang ${ }^{1}$, Anna Condylios $^{1}$, Zin Naing ${ }^{1}$, Jane-Phan $\mathrm{Au}^{1,2}$, Mariana Ruiz da Silva ${ }^{1,2}$, Charles Foster ${ }^{1,2}$

${ }^{1}$ Serology and Virology Division, NSW Health Pathology Microbiology, SEALS Prince of Wales Hospital, NSW, Australia; ${ }^{2}$ Schools of Women's and Children's Health, Medicine and Biotechnology and Biomolecular Sciences, University of New South Wales, Sydney, NSW, Australia; and ${ }^{3}$ Department of Microbiology and Infectious Diseases, NSW Health Pathology, Royal Prince Alfred Hospital, NSW, Australia

Introduction: A pandemic is an epidemic occurring worldwide, crossing international boundaries and affecting many people. The spread of SARS-CoV-2 from Hubei province in China globally has led to rapid changes in our approaches to diagnosis, with rapid use of molecular methods such as whole genome sequencing (WGS) to characterise mutations, and deployment of novel therapeutics including mRNA vaccines and monoclonal antibodies.

Methods: New methods for diagnosis, including WGS, and different approaches to emerging mutations in virus variants of concern (VOC) will be discussed.

Results and conclusions: Diagnosis now uses standard molecular methods including commercial and inhouse techniques, more recently rapid antigen detection, and research into rapid smartphone based nanoparticle assays. Laboratory practices of sample pooling, reflex testing using WGS, and workflow changes are now routine.

The use of rapid WGS techniques, ${ }^{1}$ and available Australian data, ${ }^{2,3}$ have informed assessment of the accuracy and utility of these methods in the diagnostic laboratory. Assessment of changes in PCR primer binding sites, and of the influence of how sequence data are assessed (Foster et al., in press) means accreditation and QA programs need to be continuously updated. References

1. Bull RA, Adikari TN, Ferguson JM, et al. Analytical validity of nanopore sequencing for rapid SARS-CoV-2 genome analysis. Nat Commun 2020; 11: 6272.

2. Rockett RJ, Arnott A, Lam C, et al. Revealing COVID-19 transmission in Australia by SARS-CoV-2 genome sequencing and agent-based modeling. Nat Med 2020; 26: $1398-404$

3. Seemann T, Lane CR, Sherry NL, et al. Tracking the COVID19 pandemic in Australia using genomics. Nat Commun 2020; 11: 4376.

\section{CORONIAL CASES: SARS-COV-2 INFECTION AND COVID-19}

$\underline{\text { Linda Iles }}$

Victorian Institute of Forensic Medicine, Melbourne, Vic, Australia

Victoria's second wave of SARS-CoV-2 infections saw a number of reports of deaths of SARS-CoV-2 positive persons made to the coroner. Whilst COVID-19 deaths are natural and do not routinely fall under the coroner's jurisdiction, these cases were brought to the coroner's attention due to deaths being unexpected and unexplained and subsequently being diagnosed as COVID-19 post-mortem, concerns around care in those dying with a known diagnosis of COVID-19, or co-incidental SARSCoV-2 infection with a cause of death unrelated to viral infection. This had a significant impact on workloads at VIFM, ranging from changes in admission procedures, extensive viral testing, and contributions to contact tracing. Aspects of the management of these cases will be discussed, along with the pathological aspects of some of these presentations.

\section{PATHOGEN SECURITY AND HUMAN BIOSECURITY FOR MICROBIOLOGISTS}

Gary Lum

Australian Government Department of Health, Canberra, ACT, Australia

The Security Sensitive Biological Agents Regulatory Scheme was implemented in 2009 after a Council of Australian Governments' report in 2006 recommending the establishment of a biological threat agent regulation and control framework. Legislative authority for the SSBA scheme is provided by the $\mathrm{Na}$ tional Health Security Act 2007.

Since the implementation of the scheme, some changes have been made, e.g., the 'List of SSBAs' has been reviewed and updated and changes have been made to the underpinning $\mathrm{Na}$ tional Health Security Regulations 2018. The scheme has also been regularly assessing the impact of SARS-CoV-2 since the beginning of the COVID-19 pandemic to determine whether it should be added to the List of SSBAs.

The Biosecurity Act 2015 has replaced the Quarantine Act 1908. The move to a human biosecurity framework has seen a shift in emphasis from compliance with difficult to manage quarantine arrangements to more flexible, risk-based biosecurity measures. Rather than quarantinable diseases, we now describe 'Listed Human Diseases'.

The Biosecurity Act 2015 is shared legislative responsibility between Australian Government departments, viz., the Department of Health and the Department of Agriculture. As part of this shared management, Health assists Agriculture in implementing the 'Biological Imports Program'. This includes the permit arrangements for importing microorganisms and other biological material into Australia. 